\title{
Long-term Outcomes of Adalimumab Therapy in Korean Patients with Ulcerative Colitis: A Hospital-Based Cohort Study
}

\author{
Eun Hye $\mathrm{Oh}^{1}$, Jeongseok Kim ${ }^{1}$, Namseok Ham ${ }^{1}$, Sung Wook Hwang ${ }^{1,2}$, Sang Hyoung Park ${ }^{1,2}$, Dong-Hoon Yang ${ }^{1}$, Jeong-Sik \\ Byeon $^{1}$, Seung-Jae Myung ${ }^{1}$, Suk-Kyun Yang ${ }^{1,2}$, and Byong Duk Ye ${ }^{1,2}$ \\ ${ }^{1}$ Department of Gastroenterology, and ${ }^{2}$ Inflammatory Bowel Disease Center, Asan Medical Center, University of Ulsan College of Medicine, \\ Seoul, Korea
}

Background/Aims: Studies on long-term outcomes of adalimumab therapy in non-Caucasian patients with ulcerative colitis (UC) are lacking. Methods: We analyzed long-term outcomes of Korean UC patients treated with adalimumab at the Asan Medical Center, Seoul, Korea. Results: Between July 2013 and October 2018, adalimumab therapy was started in a total of 100 patients with UC (65 males [65.0\%]; median age, 39.5 years [interquartile range, 23.3 to 49.8 years]; and median disease duration, 3.0 years [interquartile range, 1.0 to 7.0 years]). The median duration of adalimumab therapy was 13.5 months (interquartile range, 4.0 to 32.0 months). Eight of 100 patients (8.0\%) received induction therapy only, four (4.0\%) of whom ultimately underwent colectomy. Of 92 patients who received adalimumab maintenance therapy, 30 (30.0\%) stopped adalimumab therapy due to loss of response, and one patient (1.0\%) was lost to follow-up. Among the 92 patients who received adalimumab maintenance therapy, the cumulative proportions of patients remaining on adalimumab maintenance therapy were $70.0 \%$ at 1 year and $48.9 \%$ at 5 years. High partial Mayo score after 8 weeks of adalimumab therapy (hazard ratio [HR], 1.217; 95\% confidence interval [Cl], 1.040 to 1.425; $p=0.014$ ) and a history of exposure to two biologic agents before adalimumab therapy ( $\mathrm{HR}, 4.722 ; \mathrm{Cl}, 1.033$ to 21.586 ; $p=0.045$ ) were predictors of adalimumab discontinuation. Conclusions: Long-term outcomes of adalimumab therapy in Korean UC patients appear to be comparable to those in previously published Western studies. Furthermore, previous exposure to multiple biologic agents before adalimumab therapy and disease activity after 8 weeks of adalimumab therapy were predictors of adalimumab discontinuation. (Gut Liver 2020;14:347-356)
Key Words: Colitis, ulcerative; Adalimumab; Tumor necrosis factor-alpha; Prognosis

\section{INTRODUCTION}

The introduction of biologic agents, such as anti-tumor necrosis factor (anti-TNF) agents, has greatly changed treatment paradigm of patients with inflammatory bowel disease (IBD). ${ }^{1}$ According to a large-scaled retrospective cohort study on Korean ulcerative colitis (UC) patients, immunomodulators and antiTNF agents have become used more frequently, and from earlier time points during the disease course than before. ${ }^{2}$ At the same time, cumulative rates of colectomy have decreased over the last three decades. $^{2}$

In the ulcerative colitis long-term remission and maintenance with adalimumab-1, 2 (ULTRA-1,2) trials, adalimumab, a human monoclonal antibody against TNF- $\alpha$, has been proven to be efficacious in inducing remission at week 8 and maintaining remission at week 54 in patients with moderate to severe UC. ${ }^{3-5}$ It was also reported to reduce risks of all-cause, UC-related, and UC- or drug-related hospitalizations as well. ${ }^{6}$ Furthermore, adalimumab is self-injectable by patients and it was also reported to be safe and efficacious in patients who lost response or are intolerant to infliximab. ${ }^{7}$

Although efficacies of adalimumab in Asian UC patients were evaluated in a previous placebo-controlled trial from Japan, studies on real-life long-term outcomes of adalimumab therapy in Asian UC patients are still lacking. Therefore, the aim of this study was to assess long-term clinical outcomes of adalimumab therapy in a real-life cohort of Korean UC patients and to investigate the predictors affecting the outcomes.

\footnotetext{
Correspondence to: Byong Duk Ye

Department of Gastroenterology and Inflammatory Bowel Disease Center, Asan Medical Center, University of Ulsan College of Medicine, 88 Olympic-ro 43-gil, Songpa-gu, Seoul 05505, Korea

Tel: +82-2-3010-3181, Fax: +82-2-476-0824, E-mail: bdye@amc.seoul.kr

Received on April 23, 2019. Revised on June 8, 2019. Accepted on June 20, 2019. Published online September $19,2019$.

pISSN 1976-2283 eISSN 2005-1212 https://doi.org/10.5009/gnl19137

(c) This is an Open Access article distributed under the terms of the Creative Commons Attribution Non-Commercial License (http://creativecommons.org/licenses/by-nc/4.0) which permits unrestricted non-commercial use, distribution, and reproduction in any medium, provided the original work is properly cited.
} 


\section{MATERIALS AND METHODS}

\section{Study population}

This was a retrospective observational study of a single-center cohort at the IBD center of Asan Medical Center, a tertiary referral center in Seoul, Republic of Korea. Characteristics of the UC patient cohort of Asan Medical Center and patients registry were previously described. ${ }^{2}$ Patients were included if they were definitely diagnosed with UC and started to receive adalimumab therapy at the Asan Medical Center from July 2013 to October 2018. A definite diagnosis with UC was based on patients' clinical features, laboratory, endoscopic, radiologic and histologic findings. ${ }^{10}$ Patients were excluded if they were $<18$ years old or adalimumab therapy was indicated for other diseases, such as ankylosing spondylitis. All study subjects were previously exposed to immunomodulators or systemic corticosteroids, but failed to at least one out of those two medications before starting adalimumab therapy.

\section{Data collection}

Medical data of all UC patients were prospectively collected in our IBD patient registry and reviewed retrospectively at the time of the study. ${ }^{2}$ Data on demographic characteristics (sex, age, smoking status at diagnosis and family history of IBD in the first-degree relatives), disease characteristics (UC duration and extent at starting adalimumab therapy), therapies (previous exposure to other biologic agents at starting adalimumab therapy, concomitant immunomodulators use for more than 3 months during initial 6 months of adalimumab therapy), a previous history of appendectomy before starting adalimumab therapy, a history of cytomegalovirus colitis within 3 months before, or during adalimumab therapy, and laboratory findings at starting adalimumab therapy were collected. Cytomegalovirus colitis was defined as one or more inclusion bodies detected by hematoxylin and eosin staining and/or immunohistochemistry in accordance with the current European consensus. ${ }^{11-15}$

Primary outcome was set as adalimumab discontinuation due to loss of response to adalimumab and/or a colectomy. The rate of dose intensification (DI) was also evaluated. The study protocol was approved by the Institutional Review Board of Asan Medical Center (IRB number: 2017-0402).

\section{Adalimumab administration}

All patients received subcutaneous injections of adalimumab at a dose of $160 \mathrm{mg}$ at week 0 and $80 \mathrm{mg}$ at week 2 for induction therapy. Subsequently, depending on their clinical response, patients administered adalimumab at a dose of 40 mg every 2 weeks as maintenance therapy from week 4 . Based on the reimbursement policy of the Health Insurance Review and Assessment Service of Korea, clinical response to induction therapy was assessed after adalimumab dosing at week 8. In cases of decreased response during maintenance therapy, DI of adalimumab up to weekly administration was permitted.

\section{Statistical analysis}

Demographic and baseline characteristics were summarized using descriptive statistics. Categorical or nominal variables were described as numbers (\%) and continuous variables as medians (interquartile range [IQR]). The Kaplan-Meier analysis was used to evaluate cumulative proportions of patients remaining on adalimumab maintenance therapy. The Cox proportional hazard regression analysis was performed to find predictors associated with adalimumab discontinuation. Variables with a $\mathrm{p}<0.1$ by univariate analysis were included in the multivariate model. Statistical significance was defined as a p-value $<0.05$. All statistical analyses were performed using SPSS statistics version 23.0 for Windows (IBM Corp., Armonk, NY, USA).

\section{RESULTS}

\section{Baseline characteristics}

Between July 2013 and October 2018, adalimumab therapy was commenced on in a total of 100 UC patients at our center. Sixty-five patients (65.0\%) were males and median age at diagnosis of UC was 39.5 years (IQR, 23.3 to 49.8). Median disease duration at starting adalimumab therapy was 3.0 years (IQR 1.0 to 7.0$)$. Forty-two patients $(42.0 \%)$ were previously treated with one anti-TNF agent (37 [37.0\%] with infliximab/infliximab biosimilar and 5 [5.0\%] with golimumab) and two patients (2.0\%) were previously treated with two biologic agents (one with infliximab and golimumab; and the other with infliximab and vedolizumab). Sixty-eight patients (68.0\%) were treated with concomitant immunomodulators for more than 3 months during initial 6 months of adalimumab therapy. At starting adalimumab, extensive colitis was observed in 76\% (Table 1). Median duration of adalimumab therapy for 100 patients was 13.5 months (IQR, 4.0 to 32.0).

\section{Disease activity at week 0 and week 8}

At starting adalimumab therapy, most of the patients $(\mathrm{n}=78$, 78.0\%) were having severe endoscopic activity (Mayo endoscopic subscore 3) and the rest of the patients were having moderate endoscopic activity (Mayo endoscopic subscore 2). Median partial Mayo score at starting adalimumab therapy was 6.0 (IQR, 5.0 to 7.0) (Table 1). Out of 92 patients in whom results of endoscopic evaluation after 8 weeks of adalimumab therapy were available, 27 (29.4\%) and 21 (22.8\%) showed severe (Mayo endoscopic subscore 3) and moderate (Mayo endoscopic subscore 2) endoscopic activities, respectively, while 44 (47.8\%) showed remission or mild endoscopic activity (Mayo endoscopic subscore 0-1). After 8 weeks of adalimumab therapy, median partial Mayo score decreased by 3.0 (median value at week 8 , 3.0; IQR, 1.0 to 4.0 ) 
Table 1. Baseline Characteristics of the 100 Korean Patients with UC Who Received Adalimumab Therapy

\begin{tabular}{|c|c|}
\hline Variable & Value \\
\hline Male sex & $65(65.0)$ \\
\hline Age at diagnosis, yr & $39.5(23.3-49.8)$ \\
\hline Duration of disease before starting adalimumab therapy, yr & $3.0(1.0-7.0)$ \\
\hline \multicolumn{2}{|l|}{ Smoking status at diagnosis of UC } \\
\hline Never smoker & $54(54.0)$ \\
\hline Ex-smoker & $29(29.0)$ \\
\hline Current smoker & $17(17.0)$ \\
\hline Family history of inflammatory bowel disease & $7(7.0)$ \\
\hline History of appendectomy before starting adalimumab therapy & $3(3.0)$ \\
\hline \multicolumn{2}{|l|}{ p-ANCA } \\
\hline Negative & $46(46.0)$ \\
\hline Positive & $54(54.0)$ \\
\hline \multicolumn{2}{|l|}{ ASCA IgA and IgG } \\
\hline Both negative & $55(55.0)$ \\
\hline Any one positive & $24(24.0)$ \\
\hline Any one equivocal & $11(11.0)$ \\
\hline Not checked & $10(10.0)$ \\
\hline \multicolumn{2}{|l|}{ No. of biologic agents exposed before adalimumab therapy } \\
\hline Biologic-naïve & $56(56.0)$ \\
\hline Exposed to 1 biologic agent & $42(42.0)$ \\
\hline Infliximab & $33(33.0)$ \\
\hline Infliximab biosimilar & $4(4.0)$ \\
\hline Golimumab & $5(5.0)$ \\
\hline Exposed to 2 biologic agents* & $2(2.0)$ \\
\hline Immunomodulator therapy for more than 3 mo during initial 6 mo of adalimumab therapy & $68(68.0)$ \\
\hline Thiopurines & $60(60.0)$ \\
\hline Methotrexate & $8(8.0)$ \\
\hline \multicolumn{2}{|l|}{ Disease extent at starting adalimumab therapy } \\
\hline Proctitis & 0 \\
\hline Left-sided colitis & $24(24.0)$ \\
\hline Extensive colitis & $76(76.0)$ \\
\hline \multicolumn{2}{|l|}{ Mayo endoscopic subscore at starting adalimumab therapy } \\
\hline 0 & 0 \\
\hline 1 & 0 \\
\hline 2 & $22(22.0)$ \\
\hline 3 & $78(78.0)$ \\
\hline Partial Mayo score at starting adalimumab therapy & $6.0(5.0-7.0)$ \\
\hline Leukocyte count at starting adalimumab therapy, $10^{3} / \mu \mathrm{L}$ & $7.8(5.6-9.9)$ \\
\hline \multicolumn{2}{|l|}{ Hemoglobin at starting adalimumab therapy, g/dL } \\
\hline Male & $13.0(10.5-14.4)$ \\
\hline Female & $11.2(9.6-12.2)$ \\
\hline Platelet count at starting adalimumab therapy, $10^{3} / \mu \mathrm{L}$ & $318.0(269.5-402.8)$ \\
\hline \multicolumn{2}{|l|}{ ESR at starting adalimumab therapy, $\mathrm{mm} / \mathrm{hr}$} \\
\hline Male & $26.0(13.0-43.0)$ \\
\hline Female & $30.0(14.0-43.0)$ \\
\hline Serum CRP at starting adalimumab therapy, mg/dL & $0.7(0.2-1.8)$ \\
\hline Serum albumin at starting adalimumab therapy, g/dL & $3.6(2.9-3.8)$ \\
\hline
\end{tabular}

Data are presented as number (\%) or median (interquartile range).

UC, ulcerative colitis, p-ANCA, perinuclear antineutrophil cytoplasmic antibody; ASCA, anti-Saccharomyces cerevisiae antibody; Ig, immunoglubulin; ESR, erythrocyte sedimentation rate; CRP, C-reactive protein.

*One patient was treated with infliximab and golimumab, and the other was treated with infliximab and vedolizumab before adalimumab therapy. 
Table 2. Outcomes of the 92 Korean Patients with Ulcerative Colitis Who Received Adalimumab Therapy for 8 or More Weeks

\begin{tabular}{|c|c|}
\hline Variable & Value \\
\hline Median duration of adalimumab therapy, mo & $17.0(6.0-36.0)$ \\
\hline Discontinuation of adalimumab during maintenance therapy & $30(32.6)$ \\
\hline Colectomy & $9(9.8)$ \\
\hline \multicolumn{2}{|l|}{ Switched to other biologic agents } \\
\hline Infliximab/infliximab biosimilar & $3(3.3)$ \\
\hline Golimumab & $2(2.2)$ \\
\hline Vedolizumab & $2(2.2)$ \\
\hline Non-biologic therapies (5-aminosalicylates, immunomodulators, or corticosteroids) & $10(10.9)$ \\
\hline Herbal therapy & $2(2.2)$ \\
\hline No treatment & $2(2.2)$ \\
\hline DI of adalimumab & $37(40.2)$ \\
\hline Median interval from starting adalimumab to DI, mo & $6.5(3.3-13.6)$ \\
\hline Steroid rescue therapy during adalimumab therapy & $32(34.8)$ \\
\hline Median interval from starting adalimumab to steroid rescue therapy, mo & $3.5(2.0-18.3)$ \\
\hline Colectomy & $9(9.8)$ \\
\hline Median interval from starting adalimumab to colectomy, mo & $8.0(5.0-11.0)$ \\
\hline History of cytomegalovirus colitis within 3 mo before, or during adalimumab therapy & $26(26.0)$ \\
\hline
\end{tabular}

Data are presented as median (interquartile range) or number (\%).

DI, dose intensification.

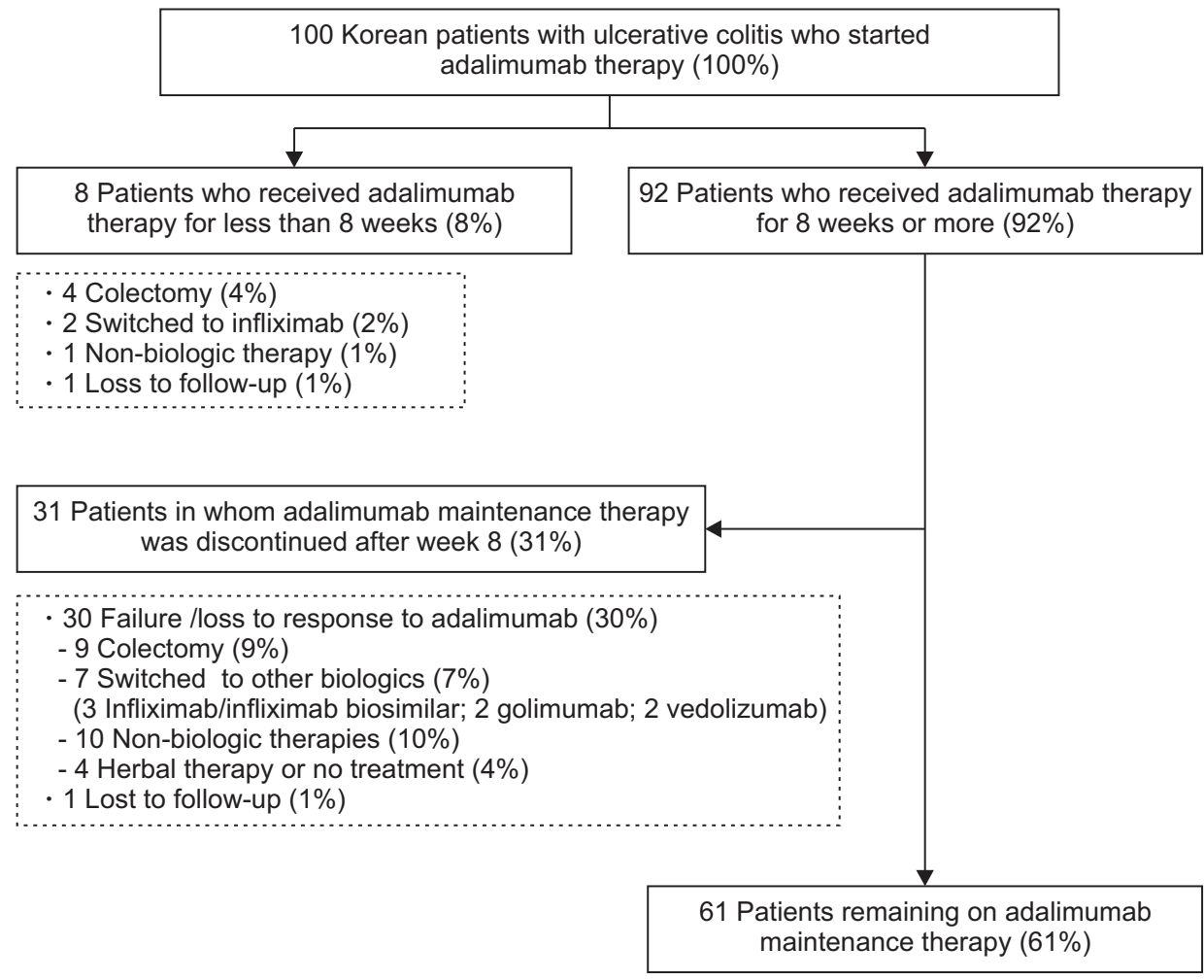

Fig. 1. Flow diagram of 100 Korean patients with ulcerative colitis who received adalimumab therapy. 


\section{Outcomes in patients who received adalimumab therapy for less than 8 weeks}

Eight out of 100 (8.0\%) patients received adalimumab therapy for less than 8 weeks. Out of eight patients, four (4.0\%) underwent colectomy and two (2.0\%) switched to infliximab. One patient (1.0\%) discontinued adalimumab after the first administration due to development of immune thrombocytopenic purpura and remained on non-biologic therapeutics (5-aminosalicylates and thiopurines) with quiescent disease. The last one (1.0\%) was lost to follow-up with active disease (Fig. 1).

\section{Outcomes in patients who received adalimumab therapy for 8 weeks or more}

Ninety-two out of 100 patients (92.0\%) received adalimumab therapy for 8 weeks or more, and median duration of adalimumab therapy among 92 patients was 17.0 months (IQR, 6.0 to 36.0). Thirty-one patients (33.7\%) stopped adalimumab maintenance therapy and 61 patients (66.3\%) were still remained on adalimumab therapy till the last follow-up (Table 2, Fig. 1).

Among 31 patients who stopped adalimumab during maintenance therapy, nine (9.8\%) underwent colectomy. Seven (7.7\%) switched to other biologic agents (three [3.3\%] to infliximab/infliximab biosimilar, two [2.2\%] to golimumab and two [2.2\%] to vedolizumab) and 10 patients (10.9\%) remained on non-biologic therapeutics (5-aminosalicylates and thiopurines). Two (2.2\%) decided to receive herbal therapy and two (2.2\%) did not receive any other treatment. The last one (1.1\%) was lost to follow-up (Table 2).

Among 92 patients with adalimumab maintenance therapy, DI was required in 37 patients (40.2\%), 6.5 months (IQR, 3.3 to 13.6) after starting adalimumab therapy. The rate of DI was calculated as 32.2\% per person-year. Thirty-two (34.8\%) required corticosteroid rescue therapy, median 3.5 months (IQR, 2.0 to 18.3) after starting adalimumab therapy (Table 2). In 20 patients, both of DI of adalimumab and corticosteroid rescue therapy were done, and corticosteroid rescue therapy preceded DI.

Cumulative proportions of remaining on adalimumab maintenance therapy was $70.0 \%, 65.8 \%, 58.1 \%$ and $48.9 \%$ at 1,2 , 3 and 5 years among 92 patients who received adalimumab therapy for 8 weeks or more (Fig. 2). When calculated among 100 patients who commenced adalimumab therapy, cumulative proportions of remaining on adalimumab maintenance therapy was $64.6 \%, 59.3 \%, 53.8 \%$ and $45.5 \%$ at 1, 2, 3 and 5 years, respectively.

\section{Predictors of adalimumab discontinuation during main- tenance therapy}

Among all variables we assessed with the Cox proportional hazard regression analysis, disease duration before starting adalimumab therapy, previous smoking at diagnosis of UC, elevated ESR, serum albumin level at the start of adalimumab therapy, partial Mayo score after 8 weeks of adalimumab therapy, previous exposure to two biologic agents and history of cytomegalovirus colitis within 3 months of, or during adalimumab therapy were included into multivariate analysis with $p<0.1$ in univariate analysis. Finally, partial Mayo score after 8 weeks of adalimumab therapy (hazard ratio [HR], 1.217; 95\% confidence interval [CI], 1.040 to $1.425 ; \mathrm{p}=0.014$ ) and previous exposure to two biologic agents (HR, 4.722; CI, 1.033 to 21.586; $\mathrm{p}=0.045$ ) turned out to be associated with adalimumab discontinuation during maintenance therapy (Table 3).

Patients with adalimumab maintenance therapy were classified into three groups according to partial Mayo score after 8 weeks of adalimumab therapy (group 1, partial Mayo score 0-2; group 2, partial Mayo score 3-5; group 3, partial Mayo score 6-9). The Kaplan-Meier analysis showed significant differences in cumulative proportions of patients remaining on adalimumab maintenance therapy among the three groups (group 1, 85.4\% and $62.0 \%$ at 1 and 4 years; group 2, 61.6\% and 38.0\% at 1 and 4 years; group 3, 36.4\% and $27.3 \%$ at 1 and 4 years; log-rank $\mathrm{p}=0.003$ ) (Fig. 3).

When patients were grouped according to the number of other biologic agents exposed before adalimumab therapy (group A, naïve to biologic agents; group $B$, exposed to one biologic agent; group $\mathrm{C}$, exposed to two biologic agents), cumulative proportions of patients remaining on adalimumab maintenance therapy significantly differed as well (group A, 78.7\% and $62.5 \%$ at 1 and 5 years; group B, 63.1\% and 37.5\% at 1 and 5 years; group $\mathrm{C}, \mathrm{n}=2$ discontinued adalimumab therapy on 125 and 148 days after starting adalimumab, respectively; logrank test $\mathrm{p}=0.001$ ) (Fig. 4). When comparing clinical outcomes in terms of clinical response defined by decrease of Mayo score (Mayo score decrease by 3 and 30\% compared to baseline)

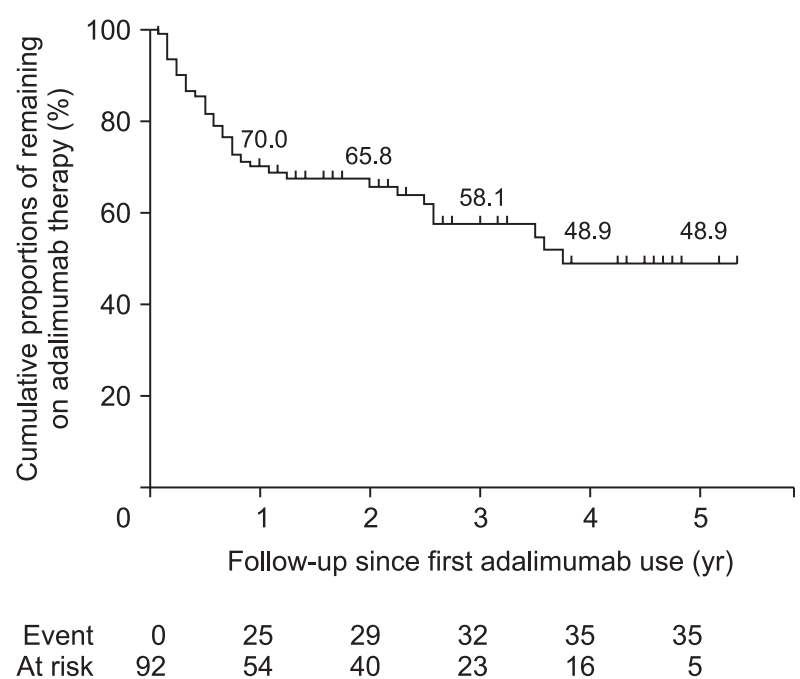

Fig. 2. Cumulative proportions of patients remaining on adalimumab therapy among 92 patients who received adalimumab therapy for 8 or more weeks. 
Table 3. Cox Regression Analysis: Predictors of Adalimumab Discontinuation in 92 Korean Patients with UC

\begin{tabular}{|c|c|c|c|c|}
\hline \multirow{2}{*}{ Variable } & \multicolumn{2}{|l|}{ Univariate analysis } & \multicolumn{2}{|c|}{ Multivariate analysis } \\
\hline & HR (95 CI) & p-value & HR (95 CI) & p-value \\
\hline Male sex & $1.102(0.540-2.253)$ & 0.789 & Not included & \\
\hline Median age at diagnosis, yr & $1.017(0.993-1.042)$ & 0.170 & Not included & \\
\hline Duration of disease before starting adalimumab therapy, yr & $0.919(0.838-1.008)$ & $0.075^{*}$ & $0.995(0.987-1.003)$ & 0.251 \\
\hline Never smoker & Reference & & & \\
\hline Ex-smoker & $1.883(0.925-3.836)$ & $0.081^{*}$ & $0.775(0.356-1.687)$ & 0.520 \\
\hline Current smoker & $0.883(0.278-2.495)$ & 0.745 & & \\
\hline Family history of inflammatory bowel disease & $0.848(0.202-3.557)$ & 0.821 & Not included & \\
\hline History of appendectomy before starting adalimumab therapy & $0.047(0.001-263.152)$ & 0.488 & Not included & \\
\hline ASCA positivity & $1.734(0.827-3.633)$ & 0.145 & Not included & \\
\hline Immunomodulator therapy for more than & $0.800(0.398-1.609)$ & 0.532 & Not included & \\
\hline \multicolumn{5}{|l|}{3 mo during initial 6 mo of adalimumab therapy } \\
\hline Disease extent at starting adalimumab therapy & & & Not included & \\
\hline Left-sided colitis & Reference & & & \\
\hline Extensive colitis & $0.826(0.386-1.767)$ & 0.623 & & \\
\hline Mayo endoscopic subscore at starting adalimumab therapy & & & Not included & \\
\hline 2 & Reference & & & \\
\hline 3 & $0.633(0.303-1.323)$ & 0.224 & & \\
\hline Partial Mayo score at starting adalimumab therapy & $1.098(0.864-1.394)$ & 0.446 & Not included & \\
\hline Leukocyte count at starting adalimumab therapy, $10^{3} / \mu \mathrm{L}$ & $1.036(0.968-1.108)$ & 0.311 & Not included & \\
\hline \multicolumn{5}{|l|}{ (<13 g/dL in male, $<12 \mathrm{~g} / \mathrm{dL}$ in female) } \\
\hline Platelet count at starting adalimumab therapy, $10^{3} / \mu \mathrm{L}$ & $1.000(0.997-1.003)$ & 0.900 & Not included & \\
\hline Elevated ESR at starting adalimumab therapy & $5.472(1.310-22.848)$ & $0.020^{*}$ & $4.324(0.985-18.988)$ & 0.052 \\
\hline \multicolumn{5}{|l|}{ (>9 mm/hr in male, $>20 \mathrm{~mm} / \mathrm{hr}$ in female) } \\
\hline Serum CRP at starting adalimumab therapy, mg/dL & $1.065(0.954-1.190)$ & 0.261 & Not included & \\
\hline Serum albumin at starting adalimumab therapy, g/dL & $0.637(0.388-1.045)$ & $0.074^{*}$ & $0.778(0.436-1.389)$ & 0.396 \\
\hline Mayo endoscopic subscore after 8 wk of adalimumab therapy & & & Not included & \\
\hline 0 & Reference & & & \\
\hline 1 & $0.708(0.237-2.116)$ & 0.537 & & \\
\hline 2 & $1.367(0.540-3.461)$ & 0.509 & & \\
\hline 3 & $1.870(0.806-4.336)$ & 0.145 & & \\
\hline Partial Mayo score after 8 wk of adalimumab therapy & $1.284(1.118-1.474)$ & $<0.001^{*}$ & $1.217(1.040-1.425)$ & $0.014^{\dagger}$ \\
\hline \multicolumn{5}{|l|}{ No. of biologic agents exposed before adalimumab therapy } \\
\hline Biologic-naïve & Reference & & & \\
\hline Exposed to 1 biologic agent & $1.753(0.878-3.497)$ & 0.111 & & \\
\hline Exposed to 2 biologic agents & $11.664(2.418-56.268)$ & $0.002^{*}$ & $4.722(1.033-21.586)$ & $0.045^{\dagger}$ \\
\hline Dose intensification of adalimumab & $1.483(0.762-2.886)$ & 0.246 & Not included & \\
\hline $\begin{array}{l}\text { History of cytomegalovirus colitis within } 3 \text { mo before, } \\
\text { or during adalimumab therapy }\end{array}$ & $2.920(1.500-5.684)$ & $0.002^{*}$ & $1.786(0.787-4.054)$ & 0.165 \\
\hline
\end{tabular}

UC, ulcerative colitis; HR, hazard ratio; CI, confidence interval; p-ANCA, perinuclear antineutrophil cytoplasmic antibody; ASCA, anti-Saccharomyces cerevisiae antibody; ESR, erythrocyte sedimentation rate; CRP, C-reactive protein.

*Variables included in multivariate analysis; ${ }^{\dagger}$ Significant predictors of adalimumab discontinuation. 


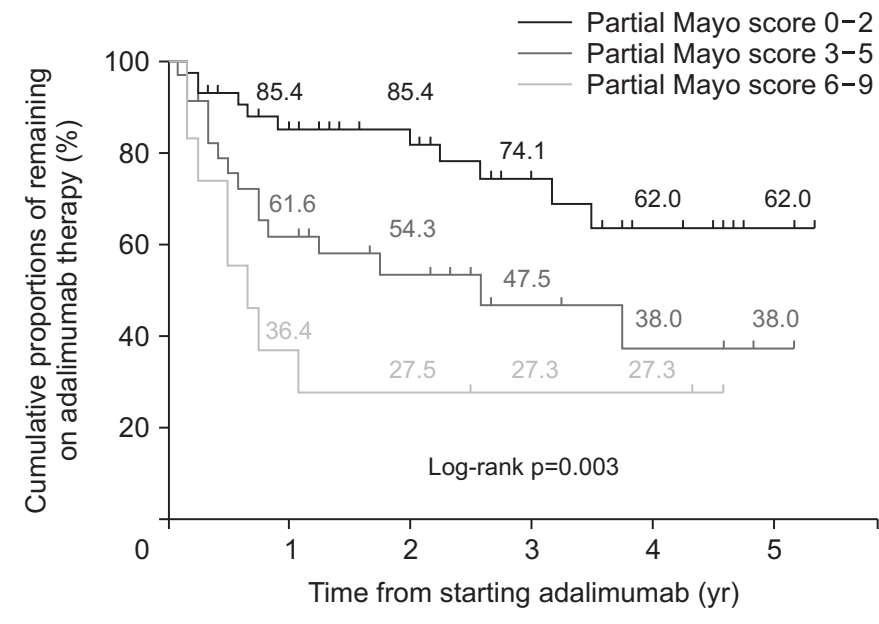

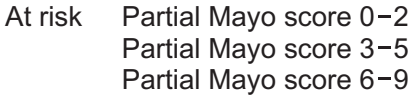

$\begin{array}{rrrrrr}45 & 32 & 25 & 15 & 10 & 3 \\ 35 & 19 & 13 & 7 & 5 & 3 \\ 12 & 5 & 4 & 3 & 3 & 0\end{array}$

Fig. 3. Cumulative proportions of patients remaining on adalimumab therapy among 92 patients who received adalimumab therapy for 8 or more weeks stratified by partial

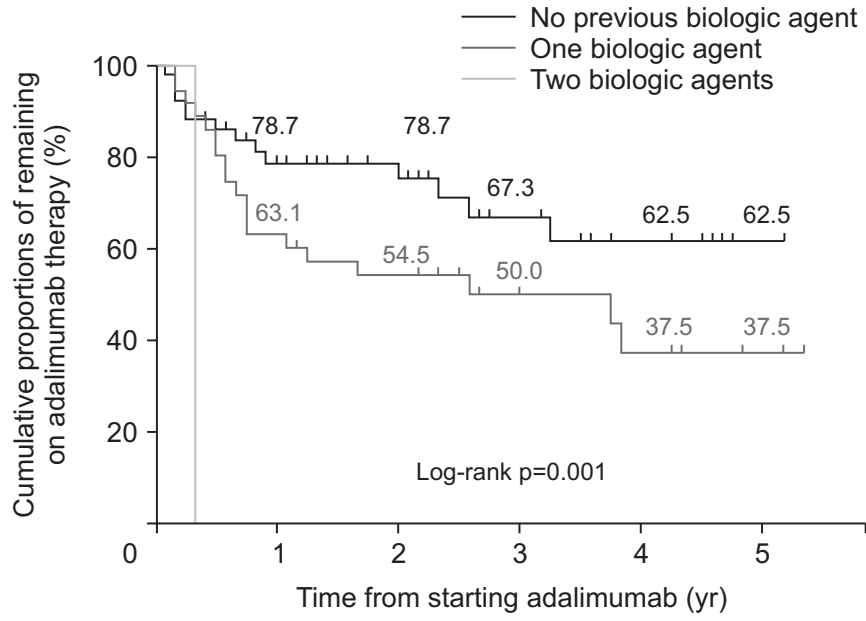

At risk No previous biologic agent One biologic agent Two biologic agents

53
37

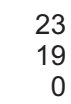

23
19
0

0

$\begin{array}{rrr}15 & 10 & 2 \\ 9 & 7 & 4 \\ 0 & 0 & 0\end{array}$

umab therapy. and clinical remission (Mayo score 0-2), biologic-naïve patients (group A) showed better clinical outcomes than biologicexposed patients (groups B and C). After 8 weeks of therapy, 46 out of 53 patients in group A (86.8\%) and 25 out of 37 patients in groups B and C (67.6\%) achieved clinical response, respectively ( $\mathrm{p}=0.046)$. Also, 29 out of 53 patients in group A (54.7\%) and 5 out of 37 patients in groups B and C (13.5\%) achieved clinical remission $(\mathrm{p}<0.001)$, respectively. At 1 year of therapy (between 50 and 55 weeks after adalimumab commencement), all 46 patients in whom Mayo score was assessed achieved clinical response. However, 18 out of 27 patients (66.7\%) in group A and 3 out of 19 patients (15.8\%) in groups B and C achieved clinical remission $(\mathrm{p}=0.001)$, respectively.

\section{DISCUSSION}

In this study, we analyzed long-term clinical outcomes of adalimumab therapy in non-Caucasian patients with UC. This is the first long-term study on a real-life cohort of Asian UC patients treated with adalimumab. In our study, more than $90 \%$ received adalimumab maintenance therapy and two-thirds out of patients entering maintenance therapy were still on adalimumab at the last follow-up. Cumulative proportions of patients remaining on adalimumab therapy was $70.0 \%$, 65.8\%, 58.1\% and $48.9 \%$ at $1,2,3$ and 5 years among 92 patients who received adalimumab maintenance therapy.

Adalimumab is the first recombinant fully human monoclonal antibody that can be self-injected subcutaneously by patients. According to Western real-life studies, it has been proven 
to be effective in treating patients with UC. In a prospective multicenter study from Hungary with 73 patients with active UC who were refractory to conventional therapy $(67.1 \%$ of the patients were previously exposed to infliximab), 75.3\% of the patients showed a short-term clinical response at week $12 .{ }^{16}$ Regarding the long-term clinical outcomes, adalimumab therapy was maintained in 48.6\% with a continuous clinical response at week $52 .{ }^{16}$ In an Italian study, the cumulative probability of continuing adalimumab therapy at 12 months was reported to be $65.9 \%$ among 88 patients with UC $(78.4 \%$ of the patients were previously exposed to infliximab). ${ }^{17}$ In a retrospective Irish study, rates of clinical response and remission were 52\% and $42 \%$, respectively among 52 UC patients (13\% of the patients were previously exposed to infliximab) at 12 months. ${ }^{18}$ In our study, because we focused on retention rate of adalimumab rather than clinical response or remission rate, it is difficult to directly compare our results with those from Hungarian and Irish studies. ${ }^{16,18}$ However, when we calculated cumulative adalimumab retention rate at 12 months among all 100 patients who commenced adalimumab therapy like the previous Italian study, the rates were very similar between two studies $(64.6 \%$ vs $65.9 \%) .{ }^{17}$ Limited option after stopping adalimumab therapy and reimbursement for life-long maintenance therapy of adalimumab with a 10\% co-payment may have influenced on the relatively high retention rate of adalimumab in our study. A substantial rate of dose escalation (40.2\%), which was similar with $35.2 \%$ from the Italian study could explain high durability of adalimumab therapy as well. ${ }^{17}$

There have been no sufficient real-life studies on the clinical benefit of adalimumab in Asian patients with UC. A placebocontrolled trial conducted in Japan showed that 50\% response rate at week 8 of adalimumab therapy among anti-TNF-naïve patients with moderate to severe UC. ${ }^{8,9}$ In another prospective study from Japan, 73.3\% of UC patients responded to adalimumab therapy at week 14 in 15 anti-TNF-naïve UC patients. ${ }^{19}$ Furthermore, the drop-out rate before week 54 was 40\% (6/15) and the response, remission, and mucosal healing rates at week 54 were 66.7\%, 53.3\%, and 58.3\%, respectively. ${ }^{19}$ In our study, 92\% of patients entered into adalimumab maintenance therapy and the cumulative rate of maintaining adalimumab therapy until 1 year was 70.0\% among the patients who received adalimumab therapy for 8 weeks or more. These figures seem to be higher when comparing with the results presented by the previously mentioned Japanese studies. ${ }^{8,9,19}$ However, this difference might be brought about because of different outcome definitions. Furthermore, as our study was conducted in reallife setting, assessment of clinical response might have been less stringent compared to a placebo-controlled trial and a prospective study from Japan. ${ }^{8,9,19}$

Partial Mayo score after 8 weeks of adalimumab therapy was found to be associated with discontinuing maintenance therapy in our study. Likewise, a previous Spanish study reported that a lack of response at week 12 was associated with an increased probability of discontinuing adalimumab and a higher rate of colectomy. ${ }^{20}$ Another study also suggested that failure of adalimumab treatment within first 3 months is associated with colectomy, ${ }^{21}$ proposing early response to adalimumab as a factor associated with a long-term durability of adalimumab.

The other factor which was related to adalimumab discontinuation in our study was previous exposure to two biologic agents. In the ULTRA-2 study, rates of clinical remission at weeks 8 and 52 were 21.3\% and 22\% in anti-TNF agent-naïve patients, while $9.2 \%$ and $10.2 \%$ in previously anti-TNF-exposed patients. ${ }^{5}$ Another study from Belgium reported that prior failure to infliximab was negatively associated with short-term mucosal healing (assessed between weeks 8 and 14). ${ }^{22}$ However, in the Hungarian study, previous infliximab therapy did not influence disease outcome and a need for DI. ${ }^{16}$ In the Italian study, previous immunosuppressant use was associated with a lower probability of clinical remission at week 54, however, previous infliximab use was not associated with clinical remission at week $54 .{ }^{17}$ In the Irish study, a trend towards better outcomes with adalimumab therapy in patients previously exposed to anti-TNF than in anti-TNF-naïve patients was observed. ${ }^{18}$ However, higher proportion of DI in anti-TNF exposed group than anti-TNF-naïve group (43\% vs 24\%) and small number of anti-TNF-exposed patients $(n=7)$ made it difficult to draw a firm conclusion. ${ }^{18}$ Therefore, it is still difficult to conclude that previous exposure to other biologics is associated with poor outcomes in adalimumab therapy for UC because of heterogeneity in study designs and definition of outcomes.

The major limitation of our study is its retrospective design. There might be factors affecting the patients' and clinicians' decisions which might have been controlled and monitored more strictly in a prospectively controlled design. However, this retrospective design could be a strength of the study at the same time, by reflecting real-life circumstances more accurately. Although information bias could be made by recall errors in retrospective study, it was minimized by using our center's IBD registry in which patients were enrolled and their data were updated prospectively by IBD specialists. ${ }^{2,23}$ The second limitation might be referral bias as our study was conducted in a single tertiary referral center. However, most moderate-to-severe UC patients using biologic agents are referred to tertiary hospitals and continuously followed, not referred back to primary or secondary medical institutions. Therefore, the patients in this study may sufficiently represent the general UC patient population receiving adalimumab therapy in Korea. Moreover, as all the patients received adalimumab therapy from a single specialized IBD center, relatively standardized treatment and follow-up strategies were ensured, thereby minimizing variations among treating clinicians. Lastly, therapeutic drug monitoring and fecal calprotectin monitoring were not systematically applied for our study subjects, which could have assisted therapeutic decision 
when performed.

In conclusion, long-term outcomes of adalimumab therapy in a real-life cohort of Korean UC patients appear to be similar to those in previously published Western and Asian studies. Partial Mayo score after 8 weeks of adalimumab therapy and a previous history of exposure to two other biologic agents before adalimumab therapy were found to be predictors for adalimumab discontinuation. These factors could be used for early optimization of adalimumab therapy considering their prognostic implication.

\section{CONFLICT OF INTEREST}

B.D.Y. has received a research grant from Celltrion; consulting fees from Abbvie Korea, Celltrion, Daewoong Pharma., Ferring Korea, Janssen Korea, Kangstem Biotech, Kuhnil Pharm., Shire Korea, Takeda Korea, IQVIA, Cornerstones Health, Robarts Clinical Trials Inc. and Takeda; speaking fees from Abbvie Korea, Celltrion, Janssen Korea, Shire Korea, Takeda Korea, and IQVIA. However, all of these are not relevant to this article.

\section{ACKNOWLEDGEMENTS}

This study was supported by a grant (number: 2010-0774) from the Asan Institute for Life Sciences, Seoul, Korea.

\section{AUTHOR CONTRIBUTIONS}

Study concept and design: E.H.O., B.D.Y. Data acquisition: E.H.O., J.K., N.H., S.W.H., S.H.P., D.H.Y., J.S.B., S.J.M., S.K.Y., B.D.Y. Data analysis and interpretation: E.H.O., B.D.Y. Drafting of the manuscript: E.H.O., B.D.Y. Study supervision: B.D.Y.

\section{ORCID}

Eun Hye Oh Jeongseok Kim Namseok Ham Sung Wook Hwang Sang Hyoung Park Dong-Hoon Yang Jeong-Sik Byeon Seung-Jae Myung Suk-Kyun Yang Byong Duk Ye

\section{REFERENCES}

1. Poullenot F, Laharie D. First line therapy of inflammatory bowel disease. Rev Prat 2014;64:1242-1248.

2. Lee HS, Park SH, Yang SK, et al. Long-term prognosis of ulcerative colitis and its temporal change between 1977 and 2013: a hospi- tal-based cohort study from Korea. J Crohns Colitis 2015;9:147155.

3. Reinisch W, Sandborn WJ, Hommes DW, et al. Adalimumab for induction of clinical remission in moderately to severely active ulcerative colitis: results of a randomised controlled trial. Gut 2011;60:780-787.

4. Sandborn WJ, Colombel JF, D'Haens G, et al. One-year maintenance outcomes among patients with moderately-to-severely active ulcerative colitis who responded to induction therapy with adalimumab: subgroup analyses from ULTRA 2. Aliment Pharmacol Ther 2013;37:204-213.

5. Sandborn WJ, van Assche G, Reinisch W, et al. Adalimumab induces and maintains clinical remission in patients with moderateto-severe ulcerative colitis. Gastroenterology 2012;142:257-265.

6. Feagan BG, Sandborn WJ, Lazar A, et al. Adalimumab therapy is associated with reduced risk of hospitalization in patients with ulcerative colitis. Gastroenterology 2014;146:110-118.

7. Afif W, Leighton JA, Hanauer SB, et al. Open-label study of adalimumab in patients with ulcerative colitis including those with prior loss of response or intolerance to infliximab. Inflamm Bowel Dis 2009;15:1302-1307.

8. Suzuki Y, Motoya S, Hanai H, et al. Four-year maintenance treatment with adalimumab in Japanese patients with moderately to severely active ulcerative colitis. J Gastroenterol 2017;52:10311040

9. Suzuki Y, Motoya S, Hanai H, et al. Efficacy and safety of adalimumab in Japanese patients with moderately to severely active ulcerative colitis. J Gastroenterol 2014;49:283-294.

10. Silverberg MS, Satsangi J, Ahmad T, et al. Toward an integrated clinical, molecular and serological classification of inflammatory bowel disease: report of a Working Party of the 2005 Montreal World Congress of Gastroenterology. Can J Gastroenterol 2005;19:5A-36A

11. Jang EY, Park SY, Lee EJ, et al. Diagnostic performance of the cytomegalovirus (CMV) antigenemia assay in patients with CMV gastrointestinal disease. Clin Infect Dis 2009;48:e121-e124.

12. Park SH, Yang SK, Hong SM, et al. Severe disease activity and cytomegalovirus colitis are predictive of a nonresponse to infliximab in patients with ulcerative colitis. Dig Dis Sci 2013;58:3592-3599.

13. Kim JW, Boo SJ, Ye BD, et al. Clinical utility of cytomegalovirus antigenemia assay and blood cytomegalovirus DNA PCR for cytomegaloviral colitis patients with moderate to severe ulcerative colitis. J Crohns Colitis 2014;8:693-701.

14. Rahier JF, Magro F, Abreu C, et al. Second European evidencebased consensus on the prevention, diagnosis and management of opportunistic infections in inflammatory bowel disease. J Crohns Colitis 2014;8:443-468.

15. Lee HS, Park SH, Kim SH, et al. Risk factors and clinical outcomes associated with cytomegalovirus colitis in patients with acute severe ulcerative colitis. Inflamm Bowel Dis 2016;22:912-918.

16. Bálint A, Farkas K, Palatka K, et al. Efficacy and safety of adalimumab in ulcerative colitis refractory to conventional therapy in 
routine clinical practice. J Crohns Colitis 2016;10:26-30.

17. Italian Group for the Study of Inflammatory Bowel Disease, Armuzzi A, Biancone L, et al. Adalimumab in active ulcerative colitis: a "real-life" observational study. Dig Liver Dis 2013;45:738743.

18. Hussey M, Mc Garrigle R, Kennedy U, et al. Long-term assessment of clinical response to adalimumab therapy in refractory ulcerative colitis. Eur J Gastroenterol Hepatol 2016;28:217-221.

19. Mizoshita T, Katano T, Tanida S, et al. Prospective comparison of preference and efficacy of adalimumab and infliximab for treating ulcerative colitis naive to antitumor necrosis factor therapy. Medicine (Baltimore) 2017;96:e7800.

20. Taxonera C, Estellés J, Fernández-Blanco I, et al. Adalimumab induction and maintenance therapy for patients with ulcerative colitis previously treated with infliximab. Aliment Pharmacol Ther 2011;33:340-348.

21. McDermott E, Murphy S, Keegan D, O’Donoghue D, Mulcahy H, Doherty G. Efficacy of Adalimumab as a long term maintenance therapy in ulcerative colitis. J Crohns Colitis 2013;7:150-153.

22. Papamichael K, Baert F, Tops S, et al. Post-induction adalimumab concentration is associated with short-term mucosal healing in patients with ulcerative colitis. J Crohns Colitis 2017;11:53-59.

23. Park SH, Yang SK, Park SK, et al. Long-term prognosis of Crohn's disease and its temporal change between 1981 and 2012: a hospital-based cohort study from Korea. Inflamm Bowel Dis 2014;20:488-494. 